\title{
ARTICLE \\ Extension of Intranuclear Cascade Model for Fragment Productions in Nucleon Induced Reactions
}

\author{
Hiroki IWAMOTO ${ }^{1, *}$ and Yusuke UOZUMI ${ }^{1}$ \\ ${ }^{1}$ Department of Applied Quantum Physics and Nuclear Engineering, Kyushu University \\ 744 Motooka, Nishi-ku, Fukuoka 819-0395, Japan
}

\begin{abstract}
A new intranuclear cascade model describing fragment productions (INC-FRG) has been developed. The fragment production model implemented in INC-FRG is based on a combination of the surface coalescence and knockout models. The current INC-FRG provides not only information of neutrons and protons but also that of fragments (clusters) up to ${ }^{4} \mathrm{He}$. Results of our calculation with INC-FRG are presented and compared with experimental data. As examples, comparisons for some proton induced reactions are shown in this article. The calculated fragment spectra considerably agree with experimental results. It is concluded that the cluster knockout contribution plays a key role in the fragment production spectra especially at forward angles.
\end{abstract}

KEYWORDS: intranuclear cascade, fragment production, surface coalescence, knockout, PHITS

\section{Introduction}

In recent years, accelerator-driven systems (ADS) of the transmutation of nuclear wastes and spallation neutron sources used in basic neutron sciences have attracted much interest. To establish these techniques, three-dimensional Monte Carlo (MC) transport codes have a key role in investigating those reliable estimations of secondary particle transportation, radiation heating and radiation damage of materials. In these MC transport codes, the intranuclear cascade (INC) and/or quantum molecular dynamics (QMD) models are employed as the event generators of nucleons, pions, and other hadrons up to several $\mathrm{GeV}$ range. However, the INC model cannot in principle describe fragment (or cluster) productions (e.g. deuteron, triton, ${ }^{3} \mathrm{He},{ }^{4} \mathrm{He}$, and other ions). Although the QMD model is capable of describing fragmentations during the cascade/dynamical stage, it is known that this model underestimates the experimental data.

Some modifications for fragment productions have been made in the INC calculation by Boudard et al. ${ }^{1)}$ and Letourneau et $a .^{2}{ }^{2}$, which are based on the phenomenological coalescence model, namely surface coalescence. On the other hand, it is well known that mechanisms of a fragment production are described as competition between pickup (coalescence) and knockout processes ${ }^{3)}$. This fact suggests that the knockout contribution should be also implemented in INC and/or QMD calculations.

We have, therefore, started to develop the original INC model $^{4)}$ (INC-FRG) describing fragment productions based on a combination of the surface coalescence and knockout models. In Ref. 4), we have demonstrated that the combination of these two models gives overall much better agreement than the normal INC model for ${ }^{12} \mathrm{C}$ and ${ }^{27} \mathrm{Al}$.

In the present study, we have upgraded the INC-FRG model to cover a broad range of target nuclei, and incorporated it into PHITS $^{5)}$ to perform the evaporation/fission calculation

\footnotetext{
*Corresponding author, E-mail: iwamotoh@nucl.kyushu-u.ac.jp
}

(C)Atomic Energy Society of Japan with the GEM code ${ }^{7)}$ following the intranuclear cascade reactions. The contributions of the fragment production models to fragment energy spectra are also investigated in detail.

\section{Intranuclear Cascade Model for Fragment Pro- ductions}

\section{Intranuclear Cascade Model}

The INC-FRG model is based on a time-dependent nucleon dynamics. A target nucleus consists of discrete nucleons determined by the Woods-Saxon density distribution and the Fermi gas momentum distribution in a $-45-\mathrm{MeV}$-deep square-shaped nuclear potential. The time evolution of nucleons obeys the Newtonian equation without any interaction as

$$
\frac{\mathrm{d} \boldsymbol{r}_{i}}{\mathrm{~d} t}=\frac{\boldsymbol{p}_{i}}{\sqrt{m_{i}^{2}+\boldsymbol{p}_{i}^{2}}},
$$

where $m_{i}$ is the mass of the $i$-th nucleon. Here, in order to maintain the constituent particles as the target nucleus, nucleons under the potential barrier are not allowed to move in spite of having their momentum. During the time evolution, it is assumed that two particles collide if their relative distance is smaller than a value obtained from the $N N$ cross section:

$$
d_{i j} \leq \sqrt{\frac{\sigma_{\mathrm{tot}}}{\pi}}
$$

where $\sigma_{\text {tot }}$ is the $N N$ total cross section $\left(\sigma_{\text {tot }}=\sigma_{\text {el }}+\sigma_{\text {inel }}\right)$. In the present calculation, we use the parametrization of Cugnon et al. ${ }^{6}$ for the $N N$ cross section and the angular distribution of $N N$ scattering. In the collision process, the Pauli blocking effect in the final state is taken into account.

\section{Surface Coalescence}

The procedure of the surface coalescence is roughly similar to the procedure of Boudard et al. ${ }^{1)}$ : First, when a nucleon, $i$, traverses the nuclear surface and is going to be emitted, it is tested whether it produces a preliminary cluster. The preliminary cluster production is judged whether the relative distance in phase space is close or not. The condition is given by

$$
r_{i j} p_{i j} \leq h_{0}
$$




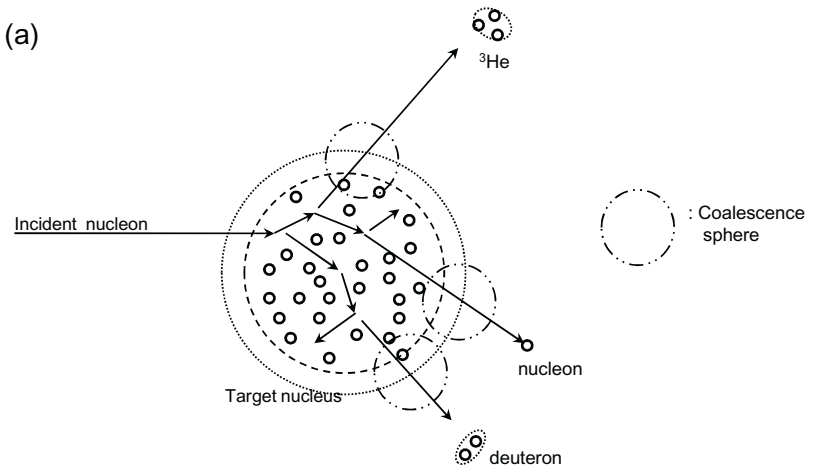

(b)

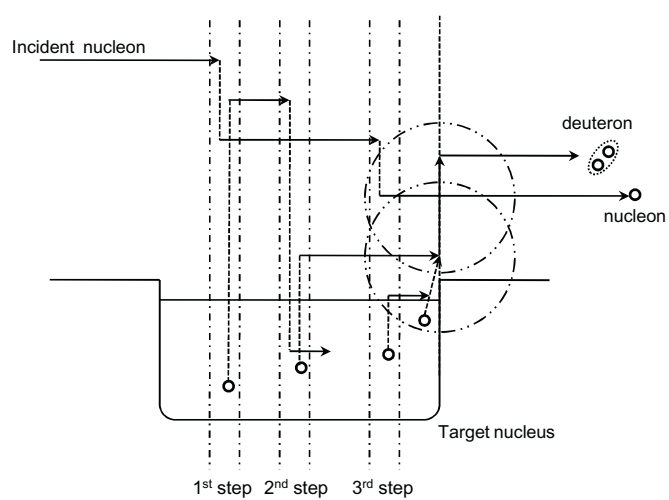

Fig. 1 Schematic representation of the surface coalescence model in $r$-space (a) and energy level diagram (b).

where $j$ denotes all other particles. $r_{i j}$ and $p_{i j}$ are defined as $r_{i j}=\left|\boldsymbol{r}_{i}-\boldsymbol{r}_{j}\right|$ and $p_{i j}=\frac{1}{2}\left|\boldsymbol{p}_{i}-\boldsymbol{p}_{j}\right|$, respectively. $h_{0}$ is the adjustable parameter to reproduce experimental data. Next, the candidate cluster is checked whether it can be raised to a realistic cluster from the isospin of the constituent nucleons. Priority of cluster productions is ordered as ${ }^{4} \mathrm{He}>\left({ }^{3} \mathrm{He}\right.$ or triton) $>$ deuteron. The center-of-mass kinetic energy of the cluster outside the nucleus is given by

$$
T_{c}=\sum_{i=1}^{A_{c}} t_{i}+V_{0}+B_{c}
$$

where $t_{i}$ is the kinetic energy of the constituent nucleon, $V_{0}$ is the potential depth, $V_{0}=-45 \mathrm{MeV}$, and $B_{c}$ denotes the binding energy of the clusters. Here the Coulomb barrier of the target nucleus is taken into account. An illustration of the surface coalescence is shown in Figure 1.

\section{Knockout}

Fragment productions by the knockout are performed according to the following procedure: When two particles collide each other, we search for nucleons around the bombarded particle in $r$-space. If there are particles in the vicinity of the nucleon, which are under the Fermi momentum, the group of nucleons is considered as a preliminary cluster. The condition is given by

$$
r_{i j} \leq r_{0}
$$

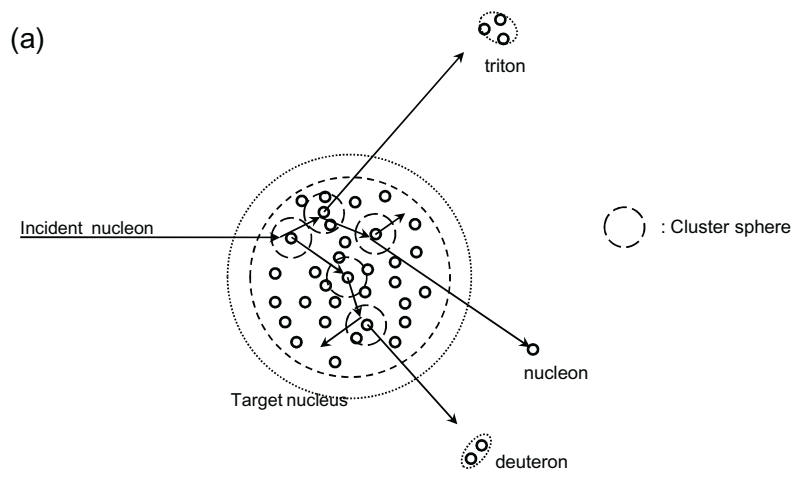

(b)

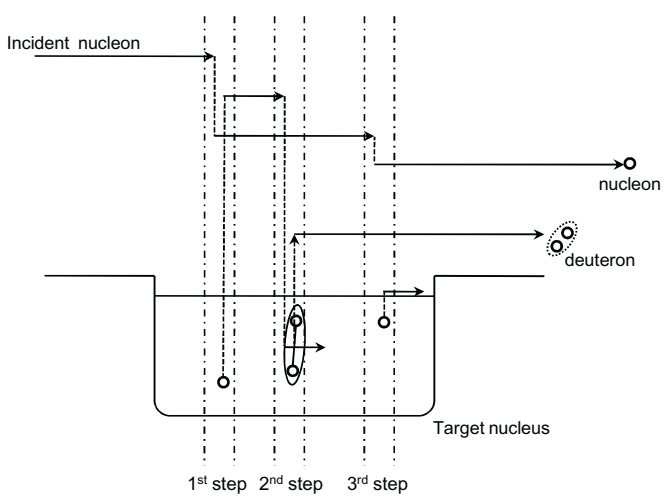

Fig. 2 Same as Figure 1 but the knockout model.

where $r_{0}$ is the free parameter corresponding to the quantity of existence of clusters. Next, we check the candidate cluster whether it can be raised to a realistic cluster in the same method as the case of the surface coalescence. Kinematics of the collision follows the $N-A N$ (nucleon-cluster) elastic scattering. The relative momenta in the center-of-momentum system of a projectile and a cluster before scattering are expressed as

$$
\boldsymbol{p}_{\mathrm{cm}}=\boldsymbol{p}_{p}+\mathscr{T} \boldsymbol{\beta}
$$

with

$$
\mathscr{T}=\gamma\left(\gamma \frac{\boldsymbol{p}_{p} \cdot \boldsymbol{\beta}}{\gamma+1}-E_{p}\right),
$$

where $\boldsymbol{\beta}$ and $\gamma$ are the boosting velocity and its gamma factor, respectively:

$$
\boldsymbol{\beta}=\frac{\boldsymbol{p}_{p}+\boldsymbol{p}_{c}}{E_{p}+E_{c}}, \quad \gamma=\frac{1}{\sqrt{1-\boldsymbol{\beta}^{2}}}
$$

where $\boldsymbol{p}_{c}$ and $E_{c}$ are given by

$$
\boldsymbol{p}_{c}=\sum_{i=1}^{A_{c}} \boldsymbol{p}_{i}, \quad E_{c}=\sqrt{\boldsymbol{p}_{c}^{2}+\left(A_{c} m\right)^{2}},
$$

respectively. Since there is no systematic data of experimental angular distribution of $N$-cluster $(A N)$ collision, we used the systematics of $N N$ collision. The center-of-mass kinetic energy of the cluster outside the nucleus $T_{c}$ is given by

$$
T_{c}=-A_{c} m+\sqrt{\boldsymbol{p}_{c}^{2}+\left(A_{c} m\right)^{2}}+V_{0}
$$



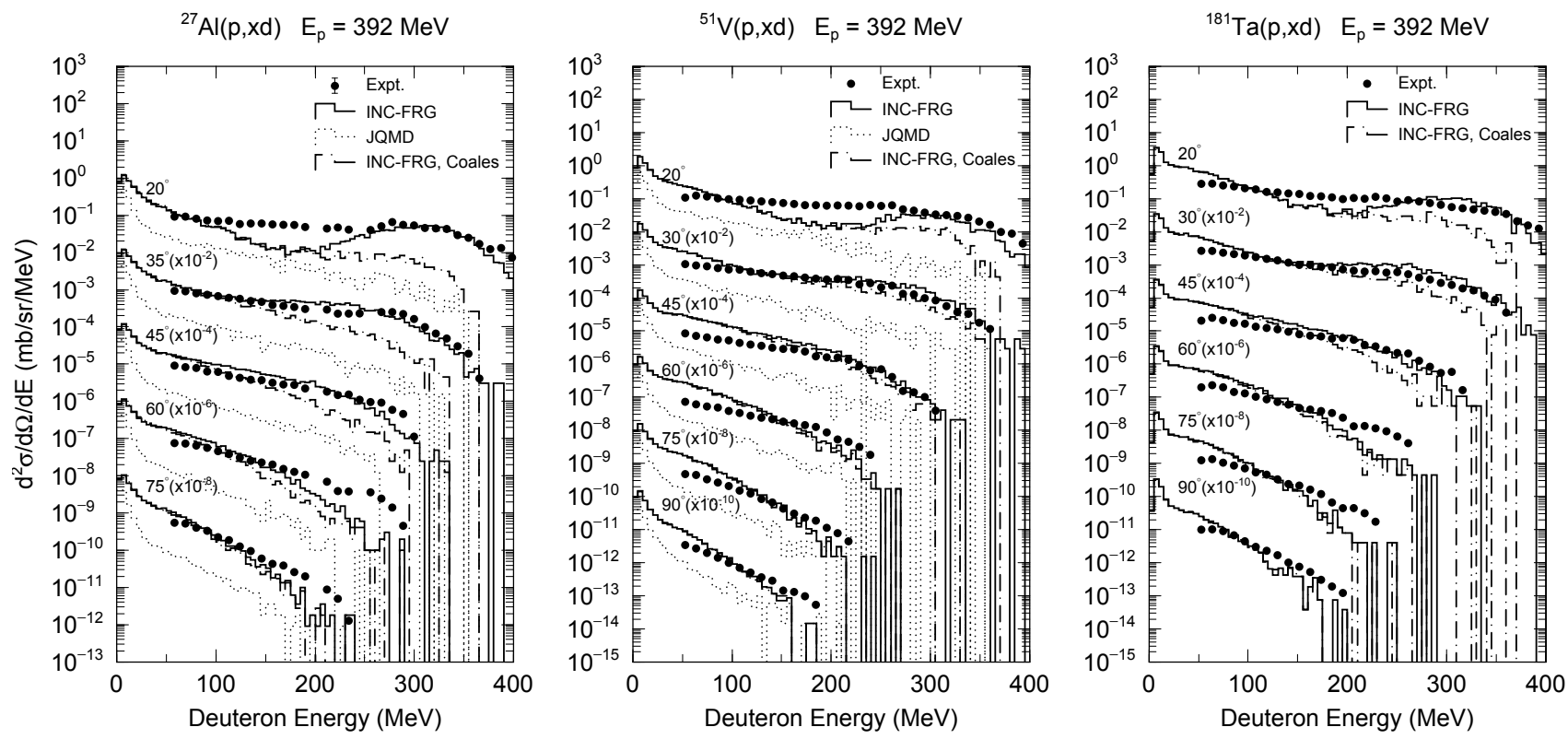

Fig. 3 Double-differential cross-sections for $392 \mathrm{MeV}$ proton on ${ }^{27} \mathrm{Al},{ }^{51} \mathrm{~V}$, and ${ }^{181} \mathrm{Ta}$. The solid and dotted histograms are the INC-FRG and JQMD calculations, respectively. The dot-dashed histograms are the INC-FRG calculation with only the surface coalescence model. The experimental data are taken from Ref. 9).

Although the cluster has a chance to collide other constituent nucleons during traveling inside the nucleus, we do not collide them any more so as to avoid complexity of the calculation. It is easy to see the knockout model from Figure 2.

\section{Results and Discussions}

\section{Deuteron Productions}

Figure 3 shows the calculated deuteron spectra for ${ }^{27} \mathrm{Al}$, ${ }^{51} \mathrm{~V}$, and ${ }^{181} \mathrm{Ta}$ compared with the experimental data together with $\mathrm{JQMD}^{8)}$. Examples of the adjustable parameters, $r_{0}$ and $h_{0}$, used in the present calculation are tabulated in Table 1. We found a considerable agreement with deuteron experimental data for the parameter sets listed in Table 1.

Table 1 Examples of adopted coalescence and knockout parameters.

\begin{tabular}{lrrrr}
\hline & ${ }^{27} \mathrm{Al}$ & ${ }^{51} \mathrm{~V}$ & ${ }^{59} \mathrm{Co}$ & ${ }^{181} \mathrm{Ta}$ \\
\hline$h_{0}(\mathrm{fm} \cdot \mathrm{MeV} / c)$ & 600 & 600 & 550 & 550 \\
$r_{0}(\mathrm{fm})$ & 0.75 & 0.50 & 0.50 & 0.50 \\
\hline
\end{tabular}

In general, the trends of JQMD results are similar to those of the surface coalescence of INC-FRG. However, JQMD underestimates the experimental deuteron spectra. One of the factors might be due to the lack of coalescence force in the two-body interaction of JQMD. In the INC-FRG model, fragments are, although phenomenologically, combined and formed by the use of the surface coalescence model. Although this coalescence model appears to be promising for a possible explanation of fragment productions, it is not enough to reach a reasonable agreement especially for forward angles.
As shown in Figure 3, this underestimate is compensated for by the knockout model, and we found that the knockout contribution increases with increasing emission energy and with decreasing target mass number. This implies that target nuclei have a cluster-like aspect together with the single particle aspect, and the cluster-like aspect plays an important role in the fragment (or cluster) emissions.

\section{Comprehensive Fragment Spectra}

As described in Sec. II, INC-FRG allows us to calculate other light fragments up to ${ }^{4} \mathrm{He}$. Figure 4 shows calculated and experimental double-differential cross-section of ${ }^{3} \mathrm{He}$ productions for $160 \mathrm{MeV}$ proton on ${ }^{59} \mathrm{Co}$ reaction. For comparison, the Bertini INC calculation is also displayed. Although INC-FRG slightly underpredicts the energy spectra, the overall trends are satisfactory.

The top panel of Figure 5 shows fragment spectra for $392 \mathrm{MeV}$ proton incident on ${ }^{27} \mathrm{Al}$ calculated by INC-FRG. It should be noted that our INC-FRG calculation predicts that the relative intensities of the different emitted particles vary by four orders of magnitude; The ratio at $300 \mathrm{MeV}$ emission energy is $\mathrm{p}: \mathrm{n}: \mathrm{d}: \mathrm{t}$ (or $\left.{ }^{3} \mathrm{He}\right): \alpha \approx 26: 7: 1: 0.03$ : 0.004 . Unfortunately, there are remarkably few fragment production data for the several hundred incident energy region. For this reason, we expect comprehensive experimental data in that energy range.

The bottom panel of Figure 5 shows comparison of calculated proton spectra between with and without the fragment production model. In Ref. 4), we have already demonstrated that our INC-FRG model without the fragment production model has a considerable predictive ability with respect to nucleon productions. Although the proton spectrum shows a de- 


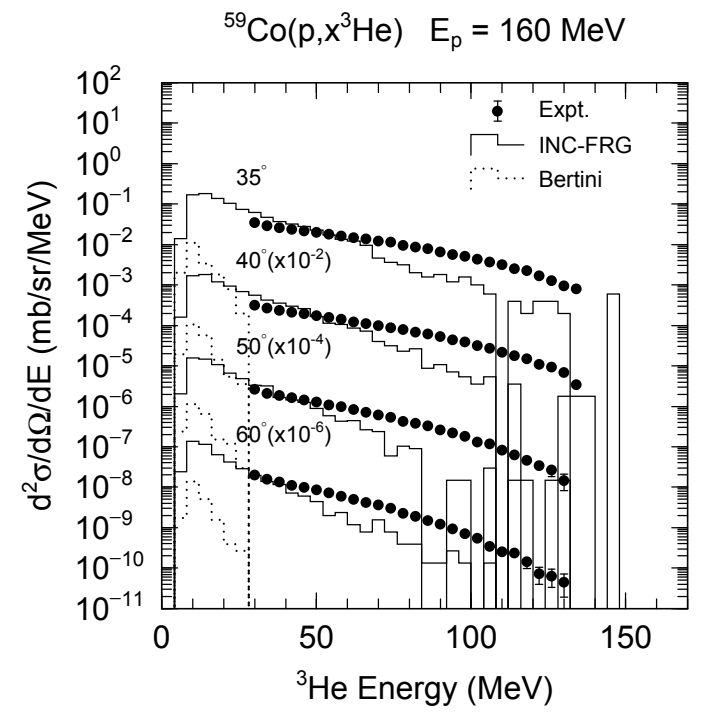

Fig. 4 Double-differential cross-sections for $160 \mathrm{MeV}$ proton incident on ${ }^{59} \mathrm{Co}$. The solid and dotted histograms are the INC-FRG and Bertini INC calculation, respectively. The experimental data are taken from Ref. 10).

crease because some protons are used in the fragment productions, it is found that this influence is small.

\section{Conclusion}

We have developed the INC model for fragment productions (INC-FRG). The fragment production model comprises the surface coalescence and knockout models. The current INC-FRG provides not only information of neutrons and protons but also that of fragments (clusters) up to ${ }^{4} \mathrm{He}$. As examples, comparisons for some proton induced reactions have been shown in this article. The calculated fragment spectra considerably agree with experimental results over a wide range of target nuclei. The cluster knockout contribution has been found to play a key role in the fragment productions especially at forward angles.

In the near future, we plan to extend the production of heavier fragments (e.g. Li, Be, B, C, etc.). It should be noted that comprehensive experimental data are essential for this extension.

\section{Acknowledgment}

The authors express their gratitude to Drs. T. Sato and D. Satoh, for their continuous encouragement and generous support. This work was supported by a Grant-in-Aid for Japan Society for the Promotion of Science (JSPS) fellows (No. 202608).

\section{References}

1) A. Boudard, J. Cugnon, S. Leray, et al., "A new model for production of fast light clusters in spallation reactions", Nucl. Phys. A 740, 195 (2004).

2) A. Letourneau, A. Böhm, J. Galin, et al., "Composite-particle emission in the reaction $p+\mathrm{Au}$ at $2.5 \mathrm{GeV}$ ", Nucl. Phys. A 717,
${ }^{27} \mathrm{Al} 392 \mathrm{MeV}(p, x p),(p, x n),(p, x d),(p, x t),(p, x h),(p, x \alpha)$
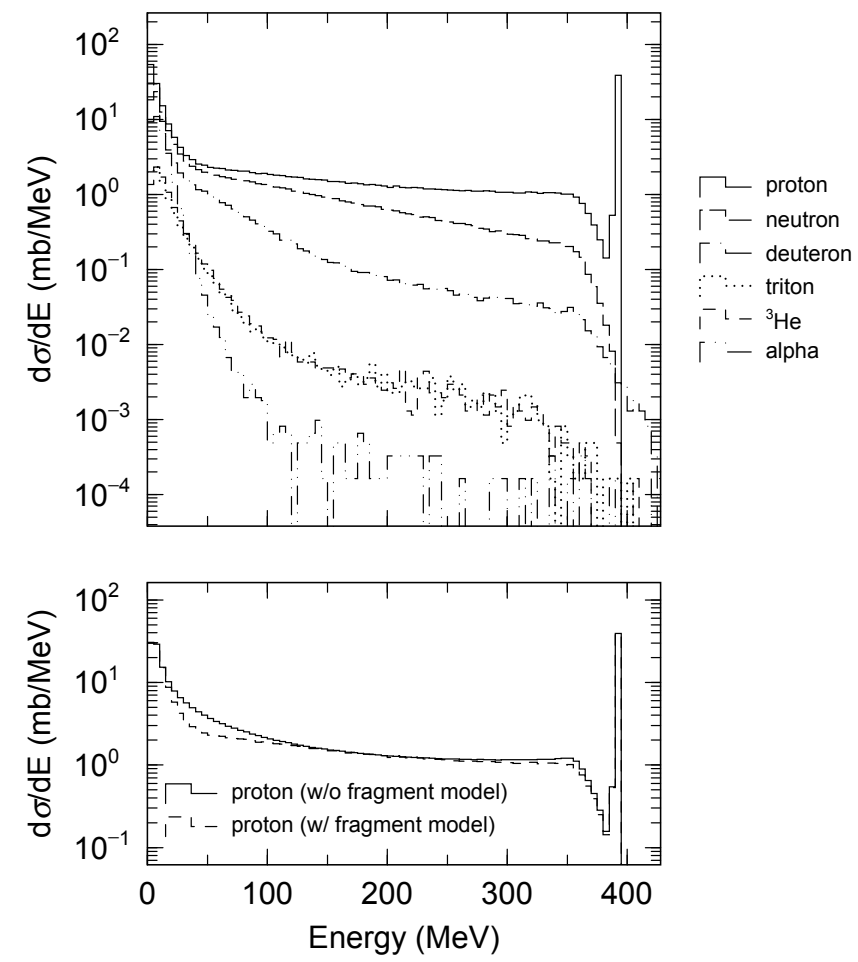

Fig. 5 (Top panel) Angle integrated fragment emission spectra for $392 \mathrm{MeV}$ proton incident on ${ }^{27} \mathrm{Al}$ calculated with INCFRG. (Bottom panel) Comparison of calculated proton spectra between with and without the fragment production model.

133 (2002).

3) P. E. Hodgson and E. Běták, "Cluster emission, transfer and capture in nuclear reactions", Phys. Rep. 374, 1 (2003).

4) H. Iwamoto and Y. Uozumi, "Cluster productions in intermediate-energy nuclear reactions," in Proceedings of the 2007 International Workshop on Compound-Nuclear Reactions and Related topics, Yosemite National Park, CA, USA, 22-26 October 2007, edited by J. Escher, F. S. Dietrich, T. Kawano, and I. Thompson, AIP Conf. Proc. 1005, 140 (2008).

5) H. Iwase, K. Niita, and T. Nakamura, "Development of a general-purpose particle heavy ion transport Monte Carlo code," J. Nucl. Sci. Technol. 39, 1142 (2002).

6) J. Cugnon, D. D'Hote, and J. Vandermeulen, "Simple parametrization of cross-section for nuclear transport studies up to GeV range", Nucl. Instr. Meth. B 111, 215 (1996).

7) S. Furihata, "Statistical analysis of light fragment production from medium energy proton-induced reactions," Nucl. Instr. Meth. B 171, 251 (2000).

8) K. Niita, S. Chiba, T. Maruyama, et al., "Analysis of the $\left(N, x N^{\prime}\right)$ reactions by quantum molecular dynamics plus statistical decay model," Phys. Rev. C52, 2620 (1995).

9) F. Saiho, Ph.D. thesis, Kyushu University, (2004) [in Japanese].

10) A. A. Cowley, J. Bezuidenhout, S. S. Dimitrova, et al., "Multistep direct mechanism in the $\left(\vec{p},{ }^{3} \mathrm{He}\right)$ inclusive reaction on ${ }^{59} \mathrm{Co}$ and ${ }^{93} \mathrm{Nb}$ at incident energies between 100 and $160 \mathrm{MeV}$," Phys. Rev. C75, 054617 (2007). 